\title{
DISCOVERY OF THE ACONITE (ACONITUM UMBROSUM (KORSH.) KOM) EVOLVEMENT STRUCTURE FROM DICOTYLEDON TO A MONOCOTYLEDON
}

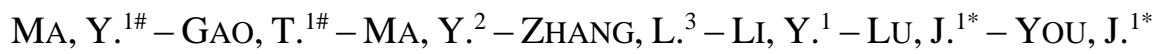 \\ ${ }^{1}$ College of Life Sciences, North East Normal University, Changchun 130024, China \\ ${ }^{2}$ School of Botany Sciences, Jilin University, Changchun 130028, China \\ ${ }^{3}$ College of College of Pharmacy, Tonghua Normal University, Tonghua 134002, China \\ ${ }^{\#}$ These authors have contributed equally to this work \\ *Corresponding authors \\ e-mail: jingmlu@163.com \\ (Received 6 ${ }^{\text {th }}$ May 2019; accepted 20 $0^{\text {th }}$ Aug 2019)
}

\begin{abstract}
An optical microscope technique and paraffin method was used to conduct an anatomical study on the stems and leaves of Aconitum umbrosum (Korsh.) Kom. (Ranunculaceae). The results showed that the stem had scattered vascular bundles, without the intrafascicular cambium. The leaf epidermis possessed pseudo-bulliform cells. The mesophyll had no distinction between palisade tissue and spongy tissue. The leaves developed multiple vascular bundles of parallel leaf veins, which had the structural characteristics of monocotyledons.

The microscopic structure of the stems and leaves of A. umbrosum, a living fossil, were analyzed as part of an attempt to confirm the evolution of plants from dicotyledons to monocots; this study contributes to the completion of the theory of evolution as seen in the Takhtajan and Cronquist systems.
\end{abstract}

Keywords: vascular bundles, vascular cylinder, bifacial leaf, isolateral leaf, mesophyll

\section{Introduction}

The genus Aconitum has about 350 species worldwide, and is widely distributed in temperate regions of the Northern Hemisphere. About 200 species of Aconitum occur in China, many of which are distributed in Northeast China. In 2019, Qin et al. through ITS sequence analysis and secondary structure prediction of 9 species of aconitum, combined with data from the public database, established a molecular method for identification of plant species of aconitum, laying a foundation for subsequent studies on authentic medicinal materials in Qinghai-Tibet Plateau (Qin et al., 2019). Li (2018) applied optical microscope and scanning electron microscope to study the phytological identification of 33 species of liliaceae belonging to 15 genera in Changbai Mountains, and summarized, sorted out and classified the phytological identification results with mathematical statistics. Tian et al. (2017) summarized aconitum plants of ranunculaceae in Qinling area from several aspects including distribution, active components, resource utilization and application of active components. Wu (2015) studied the identification and systematic evolution of epigenetic phytoliths of 17 genera of ranunculaceae, providing first-hand microscopic evidence of external morphology and internal anatomical structure. Gao (2014) described the genetic polymorphism of 7 species of aconitum from Xinjiang.

Li et al.'s (2019a), research results: WVBF and CFA may have potential synergetic effects on the target genes of certain diseases such as inflammation, cancer and diabetes. 
Li et al. (2019b) proved a preparation of two Actinomycetes plays a role in the biocontrol of root diseases and growth promotion of A. carmichaelii by inhibiting pathogen growth and shaping the rhizosphere microbiota. Xionb et al. (2019) studied the chemical constituents of the aboveground parts of aconitum in the genus ranunculaceae. Results a total of 16 compounds were isolated from dried stems and leaves of monkshood with methanol percolation. Li and Feng (1990) studied the abnormal structure of root duct group of aconite. According to Wu and Zhao (2011) there are about 200 species of aconitum in China, of which about 76 species can be used for medicinal purposes. Most aconitum medicinal plants are root tubers and roots, most of which have the effect of dispelling wind and dehumidifying, warming meridians and relieving pain, among which alkaloids are the main active ingredients. $\mathrm{Xu}$ and Dong (2009) investigated some plant resources of aconite in north China, and believed that aconite plants could be used for cut flowers, potted flowers and flower borders in gardens and had high ornamental value. Tamura et al. (1993): Dicotyledons: Magnoliid, Hamameliid and Caryophyllid.

However, there is no systematic evolutionary structure study of aconitum. This research will make efforts and contributions in this field.

\section{Materials and methods}

A. umbrosum specimens were collected in the tundra zones of Changbai Mountain in Jilin, China (Map 1). 100 samples of plant experimental materials were collected.

Permanent slides were then made as follows. First, the paraffin method was used to preserve $10-\mu \mathrm{m}$-thick slices $(\mathrm{Li}, 2018)$. Then, the slices were dehydrated by alcohol, clarified by dimethylbenzene, dyed with safranine fast green, sealed in neutral gum and observed using an Eclipse 80 microscope. Microscopic photographs were taken $(\mathrm{Wu}$, 2005).

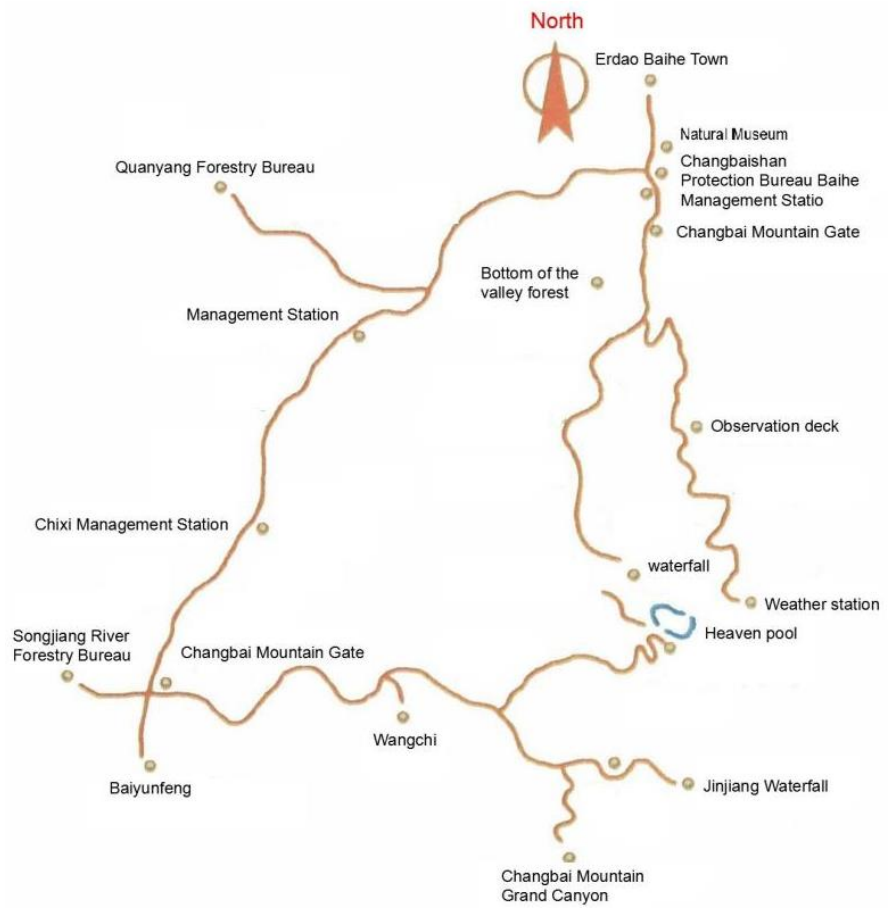

Map 1. Map of Changbai Mountain 


\section{Results}

\section{Stem anatomical structure of $A$. umbrosum}

The cross section of the stems of $A$. umbrosum was nearly round. The epidermis was composed of a single layer of tightly packed cells. Scattered heterocysts were observed, with stoma rarely seen on the epidermis (Fig. 1). In the epidermis, the epidermal cells of the external tangential wall had an average width of 2-3 $\mu \mathrm{m}$. The cortex was divided into exodermis, and into parenchyma cells of the cortex and endodermis. The thickened part of the corners of the epidermis was approximately $110 \mu \mathrm{m}$ thick. The intercellular spaces were not developed and there was an obvious thickening of the Casparian band in the endodermis. The stem had no obvious pith cell (Fig. 2).

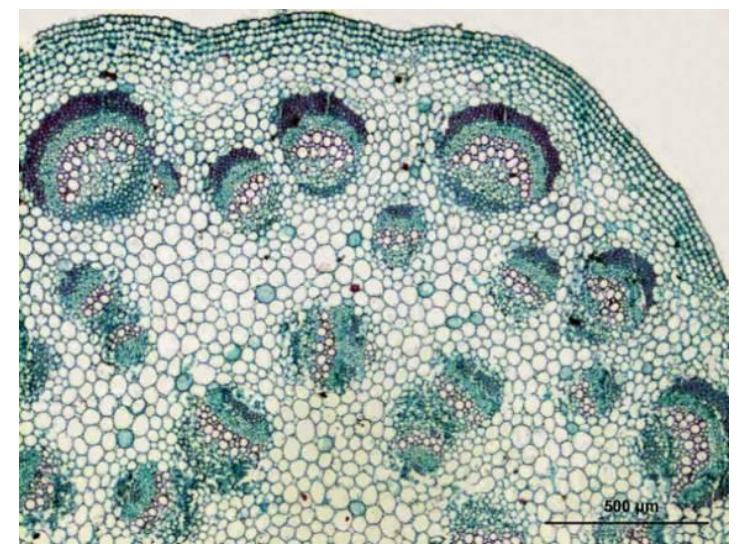

Figure 1. Cross section of stems, with somewhat scattered atactostele

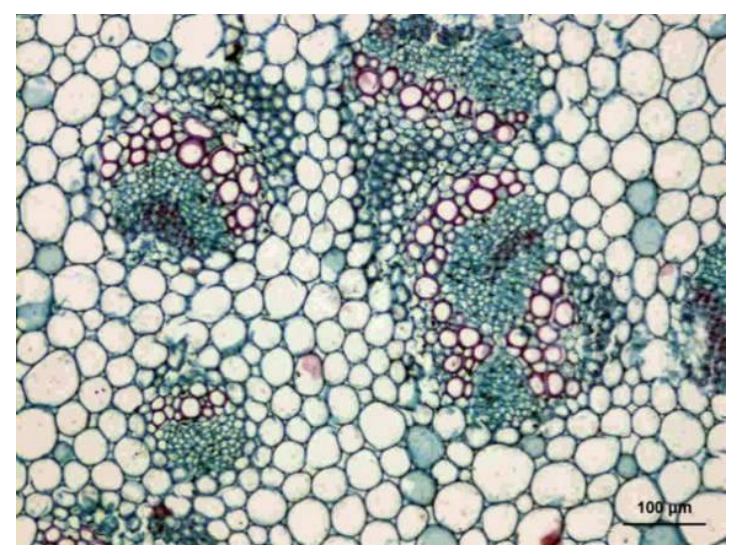

Figure 2. Cross section of stems, abnormal vascular bundles

Each stem had more than 40 vascular bundles distributed in the parenchyma tissues; bundles were either large or small in size and scattered in a disorderly fashion. The vascular bundles had obvious external contours. The vascular bundle sheath was formed by the outermost layer surrounded by sclerenchyma tissue (Figs. 3 and 4). The phloem were well-developed, composed of sieve tubes, companion cells, phloem parenchyma cells and phloem fibers. The xylem matured from the inside toward the outside. No vascular cambium was present in the bundles. 


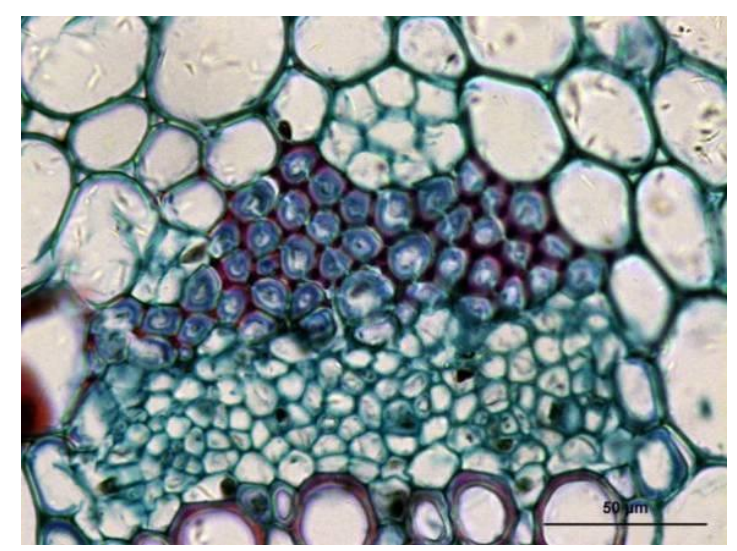

Figure 3. Cross section of stems, phloem and phloem fibers

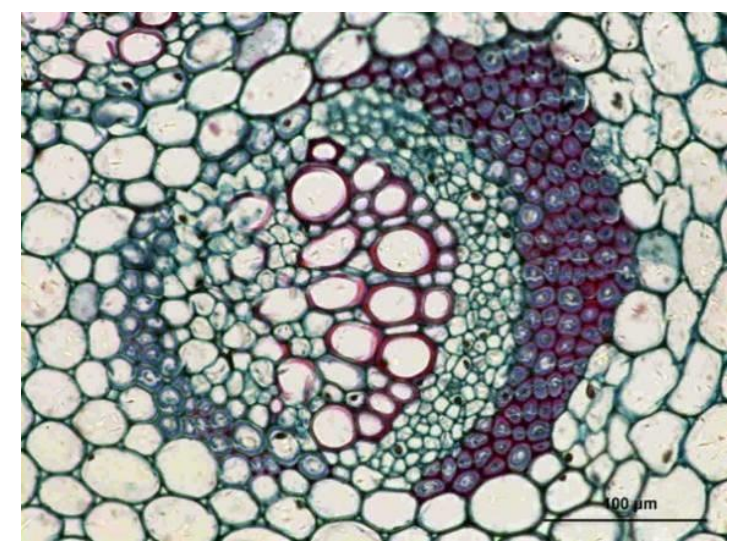

Figure 4. Cross section of stems, lack of intrafascicular cambium in vascular bundles

\section{Leaf anatomical structure of A. umbrosum}

The adaxial and abaxial epidermis was both composed of a single layer of tightly aligned cells. The epidermal cells varied in size with two or three large scattered heterocysts. The cuticle of the external tangential walls of the adaxial epidermis was more than $5 \mu \mathrm{m}$ thick. Stomata were densely distributed on the abaxial epidermis. There was no distinction between the palisade tissues and spongy tissues in the mesophyll, which had the structure of an isobilateral leaf. Cells had abundant chloroplasts and welldeveloped aerenchymas (Fig. 5 and 6 ).
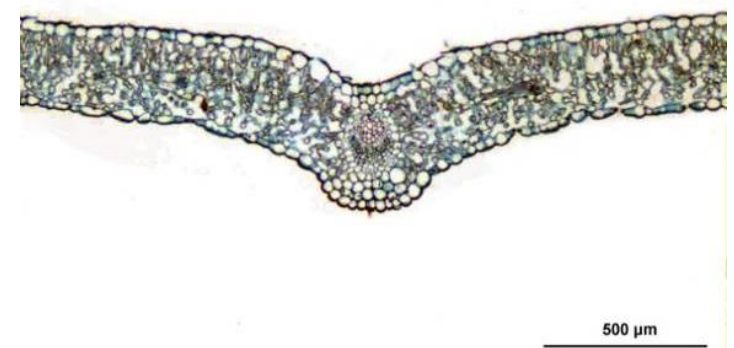

Figure 5. Cross section of a leaf, epidermis of blade, mesophyll and structure of a leaf vein 


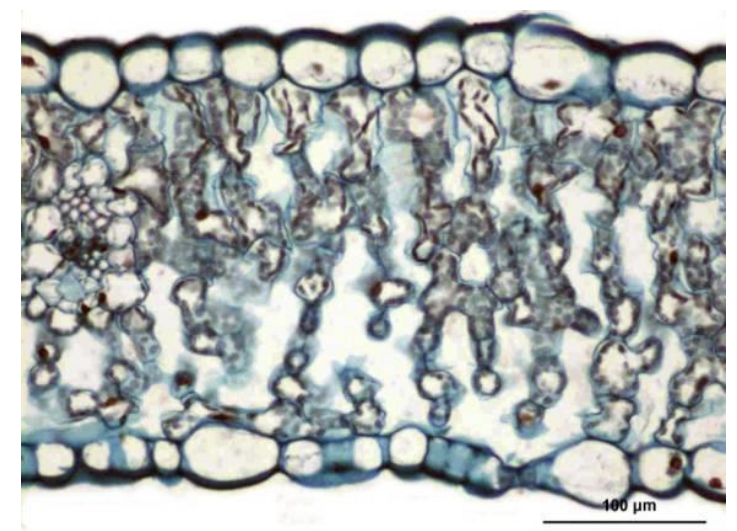

Figure 6. Cross section of a leaf, structure of an isobilateral leaf

The cross section of the main vein of the leaf was $400 \mu \mathrm{m} \times 450 \mu \mathrm{m}$. The leaf was $297 \mu \mathrm{m}$ thick. The main vein was not well developed and had few mechanical tissues. The capillaries were mostly composed of small parallel vascular bundles. The main vein was composed of phloem and xylem, without intrafascicular cambium. A layer of the vascular bundle sheath was composed of parenchyma cells that had developed around the main vein (Fig. 7). At maturity, the xylem was neither aligned on the inside nor the outside. Instead, it was aggregated in a disorderly fashion to form a special type of leaf vein. This type of vein was completely different from the structure of leaf veins of typical dicots (Fig. 8).

\section{Discussion}

The stele of the vascular plants includes protostele, siphonostele, dictyostele, eustele and atactostele, etc. (Liu, 2008). The protostele is the most primitive, while the atactostele is well-developed in monocots.

The scattered vascular bundles in the stems of A. umbrosum look similar to the atactostele of monocots. However, the atactostele of monocots has large centripetal vascular bundles and small centrifugal vascular bundles. However, the centripetal vascular bundles were small in A. umbrosum while the centrifugal ones were large. The abnormal vascular bundles for $A$. umbrosum can be called scattered atactostele-like bundles. The scattered atactostele-like bundles provide strong evidence of the evolution of A. umbrosum from dicot to monocot, which also verified the viewpoint proposed by Tamura (1964) that the Ranales represent a living ancestor of the monocots.

The vascular bundles in the stem of $A$. umbrosum had sheaths composed of sclerenchyma, with a structure similar to that of the vascular bundle sheaths in the stem of monocots. The vascular bundles in the stem had no intrafascicular cambium, which is the same as the closed vascular bundles of monocots.

The scattered distribution of vascular bundles, the vascular bundle sheath of sclerenchyma and the lack of intrafascicular cambium are typical structures of monocots. The same structures were found in this investigation in the stems of a dicot (A. umbrosum) for the first time.

The epidermal cells on the top of the leaves of A. umbrosum had obvious heterocysts with a structure similar to that of bulliform cells in the adaxial epidermis of monocots. This structure provided microscopic proof for the evolution of dicots from Ranales to 
monocots. The leaves of dicots are typically net-veined, while those of monocots have parallel veins. The structure of the pseudo-parallel veins of $A$. umbrosum is not like the netted veins of dicots. Instead, they are extremely similar to the parallel vein of monocots.

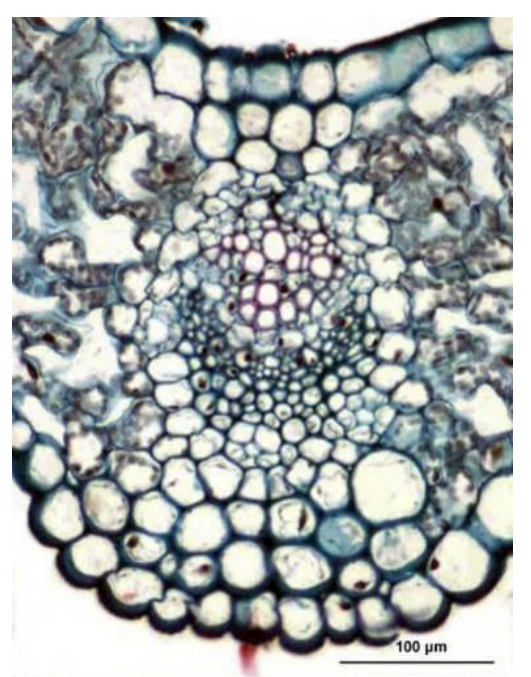

Figure 7. Vascular bundle sheath of a leaf, main vein composed of parenchyma cells

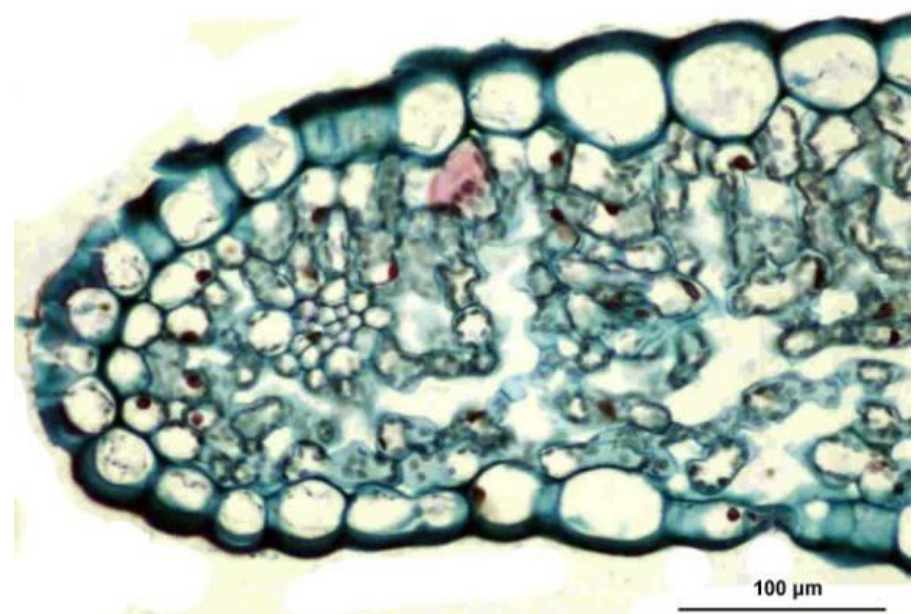

Figure 8. Cross section of a leaf, bulliform cells and heterocysts

No distinction can be seen between the palisade tissues and spongy tissues of the mesophyll of $A$. umbrosum, similar to those of the mesophyll of monocots which evolved into the structure of isobilateral leaf. There were multiple vascular bundles with small parallel leaf veins in the mesophyll in A. umbrosum; it can be considered to be a transitional type from the leaf veins of dicots (A. umbrosum) to those of monocots.

There was no intrafascicular cambium in the vascular bundles of the leaf veins, which conformed to the leaf structure of monocots. The mesophyll was not differentiated. The guard cells of the leaf stoma appeared to be transitional from the semilune type to the dumb-bell type. This type of structure has not been reported in 
dicots. This was the first time that the leaf of a dicot (A. umbrosum) was found to have a structure similar to that of monocots.

The anatomical structures of the stems and leaves of $A$. umbrosum provide microscopic proof that the study of the systematic evolution of A. umbrosum (Aconitum, Ranunculaceae) could prove useful. It also supplemented and improved the Takhtajan and the Cronquist systems of classification. Anatomical studies on the roots of $A$. umbrosum still need to be completed and discussed.

The evolutionary investigation in this article proves that the dicot (A. umbrosum) is a living fossil exhibiting the evolution from dicot to monocot.

This paper supports the idea of the evolution from dicotyledons of ranunculaceae to monocotyledons of liliaceae proposed by Tamura et al. (1993) that is, liliaceae originated from ranunculaceae.

\section{Conclusion}

The stem had scattered vascular bundles, without the intrafascicular cambium. The leaf epidermis possessed pseudo-bulliform cells. The mesophyll had no distinction between palisade tissue and spongy tissue. The leaves developed multiple vascular bundles of parallel leaf veins, which had the structural characteristics of monocotyledons. The microscopic structure of the stems and leaves of A. umbrosum, a living fossil, were analyzed as part of an attempt to confirm the evolution of plants from dicotyledons to monocots; this study contributes to the completion of the theory of evolution as seen in the Takhtajan and Cronquist systems.

This paper has obtained the first-hand experimental evidence of the evolution of dicotyledon into monocotyledon plants.

Today, we sincerely suggest that colleagues continue to carry out the structural experimental research of plant system evolution. The anatomical experiments will provide more powerful evidence of evolutionary structure for the study of plant system evolution.

Acknowledgements. This work was financially supported by The number of the national key research and development program of the 13th five-year plan, approval number: 2017YFC0803803; Project of ministry of public security, approval number:1510697607 and Changchun "double ten" science and technology project, approval number: 17SS026.

Conflicts of interests. The authors declare no conflict of interests.

\section{REFERENCES}

[1] Gao, F. C. (2014): ISSR Analysis of Genetic Polymorphism of 7 Aconite Species in Xinjiang. - Xinjiang Medical University, Xinjiang.

[2] Li, J., Liu, G., Ihsan, A., Yi, X. J., Wang, D. G., Cheng, H., Muhammad, A., Huang, X. J. (2019a): Effects of veratrilla baillonii extract on hepatic gene expression profiles in response to aconitum brachypodum-induced liver toxicity in mice. - Frontiers in Pharmacology. DOI: 10.3389/fphar.2019.00568.

[3] Li, M., Feng, Y. X. (1990): Abnormal structure of aconitum root vessel group. - Journal of Integrative Plant Biology 9: 670-673 + 742 .

[4] Li, Y. (2018): Phytological identification and phylogenetic structure of some liliaceae plants. - Doctoral Dissertation, Northeast Normal University. 
[5] Li, Y. L., Guo, Q., He, F., Li, Y. Z., Xue, Q. H., Lai, H. X. (2019b): Biocontrol of root diseases and growth promotion of the tuberous plant Aconitum carmichaelii induced by actinomycetes are related to shifts in the rhizosphere microbiota. - Microbial Ecology. DOI: 10.1007/s00248-019-01388-6.

[6] Qin, R., Zhang, D. D., Liu, H., Wu, Z. H., Zhang, J. J., Han, D., Wang, J. (2019): Sequence and structural characteristics of ITS barcodes of aconitum. - Journal of Southwest University for Nationalities (Natural Science Edition) 45(02): 134-145.

[7] Tamura, M., Ranunculaceae, A., Kubitzki, K., Rohwcr, J. G., Bittrich, V. (1993): The Families and Genera of Vascular Plants. Vol. 11. Flowering Plants. Dicotyledons: Magnoliid, Hamameliid and Caryophyllid. - Springer, Berlin.

[8] Tian, W., Zhou, T. H. (2017): Research progress of aconitum resources in Qinling area. Guangdong Chemical Industry 44(12): 155-156.

[9] Wu, C., Zhao, J. (2011): Research progress of aconitum in China. - Journal of Xinjiang Medical University 34(10): 1153-1157.

[10] Wu, D. M. (2015): Identification and systematic evolution of epigenetic phytoliths in 17 genera of ranunculaceae. - Doctoral Dissertation, Northeast Normal University.

[11] Xiong, J., Liu, W. Y., He, D., Zhang, L., Yang, C. K., Luo, Q. P., Liu, J., Shen, Y. (2019b): Study on diterpene alkaloids from overground parts of Aconitum chinensis. Chinese Herbal Medicine 50(10): 2279-2284 (in Chinese with English abstract).

[12] Xu, S., Dong, B. Y. (2009): Investigation on plant resources of aconitum in North China. - Jiangsu Agric SCI (06): 421-425. 